\title{
NONLINEAR DIFFUSION IN THE KELLER-SEGEL MODEL OF PARABOLIC-PARABOLIC TYPE
}

\author{
XiAnGSHENG XU \\ Department of Mathematics \& Statistics \\ Mississippi State University \\ Mississippi State, MS 39762, USA
}

\begin{abstract}
In this paper we study the initial boundary value problem for the system $u_{t}-\Delta u^{m}=$ $-\operatorname{div}\left(u^{q} \nabla v\right), v_{t}-\Delta v+v=u$. This problem is the so-called Keller-Segel model with nonlinear diffusion. Our investigation reveals that nonlinear diffusion can prevent overcrowding. To be precise, we show that solutions are bounded as long as $m>q>0$, thereby substantially generalizing the known results in this area. Furthermore, our result seems to imply that the Keller-Segel model can have bounded solutions and blow-up ones simultaneously.
\end{abstract}

\section{INTRODUCTION}

Theoretical and mathematical modeling of chemotaxis dates back to the works of Patlak in the 1950s [16] and Keller and Segel in the 1970s [12]. The general form of the model reads:

$$
\begin{aligned}
\partial_{t} u & =\operatorname{div}\left(k_{1}(u, v) \nabla u-k_{2}(u, v) \nabla v\right)+k_{3}(u, v) \text { in } \Omega_{T} \equiv \Omega \times(0, T], \\
\partial_{t} v & =k_{c} \Delta v+k_{4}(u, v) \text { in } \Omega_{T}, \\
\frac{\partial u}{\partial \mathbf{n}} & =\frac{\partial u}{\partial \mathbf{n}}=0 \text { on } \Sigma_{T} \equiv \partial \Omega \times(0, T], \\
\left.(u, v)\right|_{t=0} & =\left(u_{0}(x), v_{0}(x)\right) \text { on } \Omega .
\end{aligned}
$$

Here $u$ denotes the cell density and $v$ is the concentration of the chemical signal. The function $k_{1}$ is the diffusivity of the cells, $k_{2}$ is the chemotactic sensitivity, $k_{3}$ describes the cell growth and death. In the signal concentration model, $k_{4}$ describes the net effect of the production and degradation of the chemical signal. As for the remaining terms in the problem, $\Omega$ is a bounded domain in $\mathbb{R}^{N}$ with $C^{1,1}$ boundary $\partial \Omega, \mathbf{n}$ the unit outward normal to $\partial \Omega$, and $T$ any positive number.

Motivated by applications, various assumptions on the given data were suggested to further simplify the model $[6,19]$. In this paper we focus our attention on the so-called nonlinear-diffusion model. In this case,

$$
k_{1}=m u^{m-1}, \quad k_{2}=u^{q}, \quad k_{3}=0, \quad k_{c}=1, \quad k_{4}=u-v,
$$

where

$$
m, q \in(0, \infty)
$$

The resulting problem is:

$$
\begin{aligned}
u_{t}-\Delta u^{m} & =-\operatorname{div}\left(u^{q} \nabla v\right) \text { in } \Omega_{T}, \\
v_{t}-\Delta v+v & =u \text { in } \Omega_{T}, \\
\frac{\partial u^{m}}{\partial \mathbf{n}} & =\frac{\partial v}{\partial \mathbf{n}}=0 \text { on } \Sigma_{T}, \\
\left.(u, v)\right|_{t=0} & =\left(u_{0}(x), v_{0}(x)\right) \text { on } \Omega .
\end{aligned}
$$

2020 Mathematics Subject Classification. Primary: 35B45, 35B65, 35Q92, 35K51.

Key words and phrases. The Keller-Segel model, chemotaxis equations, non-linear diffusion model, global existence, uniform gradient bounds. J. Differential Equations, to appear. 
It is certainly beyond the scope of this paper to give a comprehensive review for the Keller-Segel model. In this regard, we would like to refer the reader to [8, 9]. A problem similar to (1.5)-(1.8) was investigated in $[10,11,20]$ under the assumptions that $N \geq 2, m \geq 1, q \geq 1$ (note that our $q$ here is their $q-1$ ). The global existence of a weak solution was established if, in addition, $m>q+1-\frac{2}{N}$. When this inequality fails, one obtains local existence and the global existence only holds for small data. Hölder continuity and uniqueness of weak solutions were considered in [13]. Some relevance of nonlinear diffusion in chemotaxis was discussed in [2].

The objective of this paper is to show that the results in the preceding papers can be substantially improved. Before stating our results, let us define our notion of a weak solution.

Definition 1.1. We say that $(u, v)$ is a weak solution to (1.5)-(1.8) if

$$
\begin{aligned}
& u \in L^{\infty}\left(\Omega_{T}\right), u \geq 0, u^{m} \in L^{2}\left(0, T ; W^{1,2}(\Omega)\right), \\
& v \in L^{\infty}\left(0, T ; W^{1, \infty}(\Omega)\right), \quad v \geq 0
\end{aligned}
$$

and

$$
\begin{aligned}
-\int_{\Omega_{T}} u \partial_{t} \xi d x d t+\int_{\Omega_{T}} \nabla u^{m} \nabla \xi d x d t & =\int_{\Omega} u_{0} \xi(x, 0) d x+\int_{\Omega_{T}} u^{q} \nabla v \nabla \xi d x d t, \\
-\int_{\Omega_{T}} v \partial_{t} \eta d x d t+\int_{\Omega_{T}} \nabla v \nabla \eta d x d t & =\int_{\Omega} v_{0} \eta(x, 0) d x+\int_{\Omega_{T}}(u-v) \eta d x d t
\end{aligned}
$$

for each pair of smooth functions $(\xi, \eta)$ with $\xi(x, T)=\eta(x, T)=0$.

Our main result is:

Theorem 1.2 (Main theorem). Assume:

(H1) $\Omega$ is a bounded domain in $\mathbb{R}^{N}, N \geq 3$, with $C^{1,1}$ boundary $\partial \Omega$;

(H2) $u_{0} \in L^{\infty}(\Omega), v_{0} \in W^{1, \infty}(\Omega)$ with $u_{0} \geq 0, v_{0} \geq 0$;

Then there is a weak solution $(u, v)$ to (1.5)-(1.8), provided that one of the following conditions holds.

(H3) $m>q>0$;

(H4) $m>0,1 \geq q>0$, and $q+\frac{q-1}{N+1} \leq m \leq q$.

Note that (H4) allows the possibility that $m=q=1$. This is the classical Keller-Segel system, which is well known to have blow-up solutions. Thus our theorem actually implies that the KellerSegel model can have bounded solutions and blow-up ones simultaneously. As far as we know, this is the first result in this direction. Our method seems to suggest that solutions blow up as $m \rightarrow q^{+}$, while solutions remain bounded as $m \rightarrow q^{-}$with $q<1$. All the results are established under the assumption $N>2$. But it is not difficult to see that Theorem 1.2 remains true for $N=2$.

Motivated by numerical and modeling issues, the question of how blow-up of cells can be avoided has received a lot of attention. One way of doing this is to add a cross-diffusion term to the equation for $v$ [7]. A second way is to alter the cell diffusion [1]. There are other related works. See, e.g., [2] in the context of volume effects. Here we show that nonlinear diffusion can also prevent blow-up.

Throughout this paper the letter $c$ is always used to represent a positive number whose value is determined by the given data. The norm of a function in $L^{p}(\Omega)$ is denoted by $\|\cdot\|_{p, \Omega}$. The Lebesgue measure of a set $D$ in $\mathbb{R}^{N}$ is represented by $|D|$. Whenever there is no confusion, we suppress the dependence of a function on its variables, e.g., we write $u$ for $u(x, t)$.

\section{Preliminaries}

In this section we collect a few preparatory results. The first one deals with sequences of nonnegative numbers which satisfy certain recursive inequalities. 
Proposition 2.1. Let $\left\{y_{n}\right\}, n=0,1,2, \cdots$, be a sequence of positive numbers satisfying the recursive inequalities

$$
y_{n+1} \leq c b^{n} y_{n}^{1+\alpha} \text { for some } b>1, c, \alpha \in(0, \infty)
$$

If

$$
y_{0} \leq c^{-\frac{1}{\alpha}} b^{-\frac{1}{\alpha^{2}}}
$$

then $\lim _{n \rightarrow \infty} y_{n}=0$.

This proposition can be found in ([4], p.12).

The following proposition plays a key role in the proof of our main theorem. It can be viewed as a continuous version of Lemma 3.1 in $[15,17]$.

Proposition 2.2. Let $h(\tau)$ be a continuous non-negative function defined on $\left[0, T_{0}\right]$ for some $T_{0}>$ 0 . Suppose that there exist three positive numbers $\varepsilon, \delta, b$ such that

$$
h(\tau) \leq \varepsilon h^{1+\delta}(\tau)+b \text { for each } \tau \in\left[0, T_{0}\right] .
$$

Then

$$
h(\tau) \leq \frac{1}{[\varepsilon(1+\delta)]^{\frac{1}{\delta}}} \equiv s_{0} \text { for each } \tau \in\left[0, T_{0}\right]
$$

provided that

$$
\varepsilon \leq \frac{\delta^{\delta}}{(b+\delta)^{\delta}(1+\delta)^{1+\delta}} \quad \text { and } h(0) \leq s_{0}
$$

Proof. Consider the function $f(s)=\varepsilon s^{1+\delta}-s+b$ on $[0, \infty)$. Then condition (2.1) simply says

$$
f(h(\tau)) \geq 0 \text { for each } \tau \in\left[0, T_{0}\right] .
$$

It is easy to check that the function $f$ achieves its minimum value at $s_{0}=\frac{1}{[\varepsilon(1+\delta)]^{\frac{1}{\delta}}}$. The minimum value

$$
\begin{aligned}
f\left(s_{0}\right) & =\frac{\varepsilon}{[\varepsilon(1+\delta)]^{\frac{1+\delta}{\delta}}}-\frac{1}{[\varepsilon(1+\delta)]^{\frac{1}{\delta}}}+b \\
& =b-\frac{\delta}{\varepsilon^{\frac{1}{\delta}}(1+\delta)^{\frac{1+\delta}{\delta}}} .
\end{aligned}
$$

By the first inequality in $(2.3), f\left(s_{0}\right) \leq-\delta$. Consequently, the equation $f(s)=0$ has exactly two solutions $0<s_{1}<s_{2}$ with $s_{0}$ lying in between. Evidently, $f$ is positive on $\left[0, s_{1}\right)$, negative on $\left(s_{1}, s_{2}\right)$, and positive again on $\left(s_{2}, \infty\right)$. The range of $h$ is a closed interval because of its continuity, and this interval is either contained in $\left[0, s_{1}\right)$ or $\left(s_{2}, \infty\right)$ due to (2.4). The latter cannot occur due to the second inequality in (2.3). Thus the proposition follows.

Proposition 2.3. Let $v$ be the solution of the problem

$$
\begin{aligned}
v_{t}-\Delta v+v & =u \text { in } \Omega_{T}, \\
\frac{\partial v}{\partial \mathbf{n}} & =0 \text { on } \Sigma_{T}, \\
v(x, 0) & =v_{0}(x) \text { on } \Omega .
\end{aligned}
$$

If (H1) holds, then for each $p>\frac{N+2}{2}$ there is a positive number $c$ such that

$$
\sup _{0 \leq t \leq T}\|\nabla v\|_{W^{1, \infty}(\Omega)} \leq c\left\|\nabla v_{0}\right\|_{W^{1, \infty}(\Omega)}+c\|u\|_{2 p, \Omega_{T}} .
$$


Proof. We do not believe that this result is new. However, we cannot find a good reference to it. So we offer a proof here. First we obtain a local interior estimate. The boundary estimate is achieved by flattening the relevant portion of the boundary.

Now fix a point $z_{0}=\left(x_{0}, t_{0}\right) \in \Omega_{T}$. Then pick a number $R$ from $\left(0, \min \left\{\operatorname{dist}\left(x_{0}, \partial \Omega\right), \sqrt{t_{0}}\right\}\right)$. Define a sequence of cylinders $Q_{R_{n}}\left(z_{0}\right)$ in $\Omega_{T}$ as follows:

$$
Q_{R_{n}}\left(z_{0}\right)=B_{R_{n}}\left(x_{0}\right) \times\left(t_{0}-R_{n}^{2}, t_{0}\right],
$$

where

$$
R_{n}=\frac{R}{2}+\frac{R}{2^{n+1}} \quad n=0,1,2, \cdots .
$$

Choose a sequence of smooth functions $\theta_{n}$ so that

$$
\begin{aligned}
\theta_{n}(x, t) & =1 \text { in } Q_{R_{n}}\left(z_{0}\right), \\
\theta_{n}(x, t) & =0 \text { outside } B_{R_{n-1}}\left(x_{0}\right) \text { and } t<t_{0}-R_{n-1}^{2}, \\
\left|\partial_{t} \theta_{n}(x, t)\right| \leq \frac{c 4^{n}}{R^{2}} \text { on } Q_{R_{n-1}}\left(z_{0}\right), & \\
\left|\nabla \theta_{n}(x, t)\right| & \leq \frac{c 2^{n}}{R} \text { on } Q_{R_{n-1}}\left(z_{0}\right), \text { and } \\
0 & \leq \theta_{n}(x, t) \leq 1 \text { on } Q_{R_{n-1}}\left(z_{0}\right) .
\end{aligned}
$$

Let $p$ be given as in the lemma. Select

$$
K \geq R^{1-\frac{N+2}{2 p}}\|u\|_{2 p, Q_{R}\left(z_{0}\right)}
$$

as below. Set

$$
K_{n}=K-\frac{K}{2^{n+1}}, \quad n=0,1,2, \cdots .
$$

Fix an $i \in\{1, \cdot, N\}$. Define

$$
w=v_{x_{i}} .
$$

Then $w$ satisfies the equation

$$
w_{t}-\Delta w+w=u_{x_{i}} \text { in } \Omega_{T} .
$$

Without loss of generality, assume $\sup _{\Omega_{T}} w=\|w\|_{\infty, \Omega_{T}}$. We use $\theta_{n+1}^{2}\left(w-K_{n+1}\right)^{+}$as a test function in (2.12) to derive

$$
\begin{aligned}
& \frac{1}{2} \frac{d}{d t} \int_{\Omega} \theta_{n+1}^{2}\left[\left(w-K_{n+1}\right)^{+}\right]^{2} d x+\int_{\Omega} \theta_{n+1}^{2}\left|\nabla\left(w-K_{n+1}\right)^{+}\right|^{2} d x+\int_{\Omega} w \theta_{n+1}^{2}\left(w-K_{n+1}\right)^{+} d x \\
&= \int_{\Omega} \theta_{n+1} \partial_{t} \theta_{n+1}\left[\left(w-K_{n+1}\right)^{+}\right]^{2} d x-2 \int_{\Omega} \theta_{n+1} \nabla \theta_{n+1} \nabla w\left(w-K_{n+1}\right)^{+} d x \\
& \quad-\int_{\Omega} u \theta_{n+1}^{2} \partial_{x_{i}}\left(w-K_{n+1}\right)^{+}-2 \int_{\Omega} u \theta_{n+1} \partial_{x_{i}} \theta_{n+1}\left(w-K_{n+1}\right)^{+} d x,
\end{aligned}
$$

from whence follows

$$
\begin{aligned}
& \sup _{0 \leq t \leq t_{0}} \int_{\Omega} \theta_{n+1}^{2}\left[\left(w-K_{n+1}\right)^{+}\right]^{2} d x+\int_{0}^{t_{0}} \int_{\Omega} \theta_{n+1}^{2}\left|\nabla\left(w-K_{n+1}\right)^{+}\right|^{2} d x d t \\
& \quad \leq \frac{c 4^{n}}{R^{2}} \int_{Q_{R_{n}}\left(z_{0}\right)}\left[\left(w-K_{n+1}\right)^{+}\right]^{2} d x d t+c \int_{A_{n+1}} u^{2} \theta_{n+1}^{2} d x d t \\
& \quad \leq \frac{c 4^{n}}{R^{2}} y_{n}+c\left\|u^{2}\right\|_{p, Q_{R}\left(z_{0}\right)}\left|A_{n+1}\right|^{1-\frac{1}{p}}
\end{aligned}
$$


where

$$
\begin{aligned}
y_{n} & =\int_{Q_{R_{n}}\left(z_{0}\right)}\left[\left(w-K_{n}\right)^{+}\right]^{2} d x d t, \\
A_{n+1} & =\left\{(x, t) \in Q_{R_{n}}\left(z_{0}\right): w(x, t) \geq K_{n+1}\right\} .
\end{aligned}
$$

By Poincaré's inequality,

$$
\begin{aligned}
& \int_{0}^{t_{0}} \int_{\Omega}\left[\theta_{n+1}\left(w-K_{n+1}\right)^{+}\right]^{\frac{4}{N}+2} d x d t \\
& \quad \leq \int_{0}^{t_{0}}\left(\int_{\Omega}\left[\theta_{n+1}\left(w-K_{n+1}\right)^{+}\right]^{2} d x\right)^{\frac{2}{N}}\left(\int_{\Omega}\left[\theta_{n+1}\left(w-K_{n+1}\right)^{+}\right]^{\frac{2 N}{N-2}}\right)^{\frac{N-2}{N}} d t \\
& \quad \leq\left(\sup _{0 \leq t \leq t_{0}} \int_{\Omega}\left[\theta_{n+1}\left(w-K_{n+1}\right)^{+}\right]^{2} d x\right)^{\frac{2}{N}} \int_{0}^{t_{0}} \int_{\Omega}\left|\nabla\left(\theta_{n+1}\left(w-K_{n+1}\right)^{+}\right)\right|^{2} d x d t \\
& \leq c\left(1+4^{n}\right)\left(\frac{c 4^{n}}{R^{2}} y_{n}+c\left\|u^{2}\right\|_{p, Q_{R}\left(z_{0}\right)}\left|A_{n+1}\right|^{1-\frac{1}{p}}\right)^{\frac{N+2}{N}} .
\end{aligned}
$$

Subsequently,

$$
\begin{aligned}
y_{n+1} & =\int_{Q_{R_{n+1}}\left(z_{0}\right)}\left[\left(w-K_{n+1}\right)^{+}\right]^{2} d x d t \\
& \leq \int_{0}^{t_{0}} \int_{\Omega}\left[\theta_{n+1}\left(w-K_{n+1}\right)^{+}\right]^{2} d x d t \\
& \leq\left(\int_{0}^{t_{0}} \int_{\Omega}\left[\theta_{n+1}\left(w-K_{n+1}\right)^{+}\right]^{2 \frac{N+2}{N}} d x d t\right)^{\frac{N}{N+2}}\left|A_{n+1}\right|^{\frac{2}{N+2}} \\
& \leq c 4^{\frac{N n}{N+2}}\left(\frac{c 4^{n}}{R^{2}} y_{n}+c\left\|u^{2}\right\|_{p, Q_{R}\left(z_{0}\right)}\left|A_{n+1}\right|^{1-\frac{1}{p}}\right)\left|A_{n+1}\right|^{\frac{2}{N+2}} \\
& \leq c 4^{\frac{N n}{N+2}}\left(\frac{c 4^{n}}{R^{2}} y_{n}+c R^{\frac{N+2}{p}-2} K^{2}\left|A_{n+1}\right|^{1-\frac{1}{p}}\right)\left|A_{n+1}\right|^{\frac{2}{N+2}} .
\end{aligned}
$$

The last step is due to (2.10). We also have

$$
y_{n} \geq \int_{A_{n+1}}\left(K_{n+1}-K_{n}\right)^{2} d x d t=\frac{K^{2}}{4^{n+1}}\left|A_{n+1}\right| .
$$

It immediately follows that

$$
\begin{aligned}
y_{n}\left|A_{n+1}\right|^{\frac{2}{N+2}} & =y_{n}\left|A_{n+1}\right|^{\frac{1}{p}}\left|A_{n+1}\right|^{\frac{2}{N+2}-\frac{1}{p}} \\
& \leq \frac{c R^{\frac{N+2}{p}} 4^{\frac{(n+1)[2 p-(N+2)]}{p(N+2)}}}{K^{2 \frac{2 p-(N+2)}{p(N+2)}} y_{n}^{1+\frac{2 p-(N+2)}{p(N+2)}}}, \\
K^{2}\left|A_{n+1}\right|^{1-\frac{1}{p}}\left|A_{n+1}\right|^{\frac{2}{N+2}} & =K^{2}\left|A_{n+1}\right|\left|A_{n+1}\right|^{\frac{2}{N+2}-\frac{1}{p}} \\
& \leq \frac{c 4^{n+1+\frac{(n+1)[2 p-(N+2)]}{p(N+2)}} y_{n}^{1+\frac{2 p-(N+2)}{p(N+2)}}}{K^{2 \frac{2 p-(N+2)}{p(N+2)}}} .
\end{aligned}
$$

Use these in (2.18) to derive

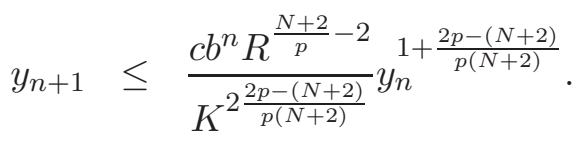


By Proposition 2.1, if we choose $K$ so large that

$$
y_{0} \leq c K^{2} R^{N+2}
$$

then

$$
\sup _{Q_{\frac{R}{2}}\left(z_{0}\right)} w \leq K
$$

In view of $(2.10)$, it is enough for us to take

$$
K=c\left(\frac{y_{0}}{R^{N+2}}\right)^{\frac{1}{2}}+R^{1-\frac{N+2}{2 p}}\|u\|_{2 p, Q_{R}\left(z_{0}\right)} .
$$

Recall that

$$
y_{0}=\int_{Q_{R}\left(z_{0}\right)}\left[\left(w-\frac{K}{2}\right)^{+}\right]^{2} d x d t \leq \int_{Q_{R}\left(z_{0}\right)}\left(w^{+}\right)^{2} d x d t
$$

Hence,

$$
\sup _{Q_{\frac{R}{2}}\left(z_{0}\right)} w \leq c\left(\underset{Q_{R}\left(z_{0}\right)}{f}\left(w^{+}\right)^{2} d x d t\right)^{\frac{1}{2}}+R^{1-\frac{N+2}{2 p}}\|u\|_{2 p, Q_{R}\left(z_{0}\right)} .
$$

This is the so-called local interior estimate. Now we proceed to derive the boundary estimate. Suppose $x_{0} \in \partial \Omega$. Our assumption on the boundary implies that there exist a neighborhood $U\left(x_{0}\right)$ of $x_{0}$ and a $C^{1,1}$ diffeomorphism $\mathbb{T}$ defined on $U\left(x_{0}\right)$ such that the image of $U\left(x_{0}\right) \cap \Omega$ under $\mathbb{T}$ is the half ball $B_{\delta}^{+}\left(y_{0}\right)=\left\{y:\left|y-y_{0}\right|<\delta, y_{i}>0\right\}$, where $\delta>0, y_{0}=\mathbb{T}\left(x_{0}\right)$, and $i$ is given as in (2.11). This implies that we have flatten $U\left(x_{0}\right) \cap \partial \Omega$ into a region in the plane $y_{i}=0$ in the $y$ space [3]. Set

$$
\tilde{v}=v \circ \mathbb{T}^{-1}, \quad \tilde{w}=\tilde{v}_{y_{i}} .
$$

We can choose $\mathbb{T}$ so that $\tilde{w}=\tilde{w}(y, t)$ satisfies the boundary condition

$$
\left.\tilde{w}\right|_{y_{i}=0}=\left.\tilde{v}_{y_{i}}\right|_{y_{i}=0}=\left.\frac{\partial \tilde{v}}{\partial \mathbf{n}}\right|_{y_{i}=0}=0 .
$$

One way of doing this is to pick $\mathbb{T}=\left(\begin{array}{c}f_{1}(x) \\ \vdots \\ f_{N}(x)\end{array}\right)$ so that the graph of $f_{1}(x)=0$ is $U\left(x_{0}\right) \cap \partial \Omega$ and the set of vectors $\left\{\nabla f_{1}, \cdots, \nabla f_{N}\right\}$ is orthogonal. By a result in [21], $\tilde{w}$ satisfies the equation

$$
\partial_{t} \tilde{w}-\operatorname{div}\left[\left(J_{\mathbb{T}}^{T} J_{\mathbb{T}}\right) \circ \mathbb{T}^{-1} \nabla \tilde{w}\right]+\tilde{w}=\left(\mathbf{h} J_{\mathbb{T}}\right) \circ \mathbb{T}^{-1} \nabla \tilde{w}+\left(J_{\mathbb{T}} \circ \mathbb{T}^{-1} \nabla \tilde{u}\right)_{i} \quad \text { in } B_{\delta}^{+}\left(y_{0}\right),
$$

where $J_{\mathbb{T}}$ is the Jacobian matrix of $\mathbb{T}$, i.e.,

$$
J_{\mathbb{T}}=\nabla \mathbb{T},
$$

$\left(J_{\mathbb{T}} \circ \mathbb{T}^{-1} \nabla \tilde{u}\right)_{i}$ is the $\mathrm{i}$-th component of the vector $J_{\mathbb{T}} \circ \mathbb{T}^{-1} \nabla \tilde{u}$, and the row vector $\mathbf{h}$ is roughly $\operatorname{div}\left(J_{\mathbb{T}}^{T} J_{\mathbb{T}}\right)$ and is, therefore, bounded by our assumption on $\mathbb{T}$. In view of $(2.28)$, the method employed to prove $(2.27)$ still works here. The only difference is that we use $B_{R_{n}}^{+}\left(y_{0}\right)$ instead of $B_{R_{n}}\left(y_{0}\right)$ in the proof. If $t_{0}=0$, then we just need to change $Q_{R_{n}}\left(z_{0}\right)$ to $B_{R_{n}} \times\left[0, R_{n}^{2}\right)$ and require

$$
K \geq 2\left\|\nabla v_{0}\right\|_{\infty, \Omega}
$$

in addition to (2.10) in the proof . Subsequently, (2.27) follows.

Finally, use $v$ as a test function in (3.14) to derive

$$
\frac{1}{2} \frac{d}{d t} \int_{\Omega} v^{2}+\int_{\Omega}|\nabla v|^{2} d x+\int_{\Omega} v^{2} d x=\int_{\Omega} u v d x .
$$


It immediately follows that

$$
\int_{\Omega_{T}}|\nabla v|^{2} d x d t \leq c \int_{\Omega_{T}} u^{2} d x d t+c \int_{\Omega} v_{0}^{2} d x
$$

The classical $L^{\infty}$ estimate for linear parabolic equations asserts

$$
\|v\|_{\infty, \Omega_{T}} \leq c\|u\|_{p, \Omega_{T}}+\left\|v_{0}\right\|_{\infty, \Omega} .
$$

This completes the proof.

\section{Proof of TheOrem 1.2}

A solution to (1.5)-(1.8) is constructed as the limit of a sequence of approximate solutions. Our approximate problems are formulated as follows (also see [20]):

$$
\begin{aligned}
\partial_{t} U-m \operatorname{div}\left(\left(U^{+}+\sigma\right)^{m-1} \nabla U\right) & =-\operatorname{div}\left(\left(U^{+}\right)^{q} \nabla V\right) \text { in } \Omega_{T}, \\
\partial_{t} V-\Delta V+V & =U \text { in } \Omega_{T}, \\
\frac{\partial U}{\partial \mathbf{n}} & =\frac{\partial V}{\partial \mathbf{n}}=0 \text { on } \Sigma_{T}, \\
\left.(U, V)\right|_{t=0} & =\left(u_{0}, v_{0}\right) \text { on } \Omega,
\end{aligned}
$$

where $\sigma>0$. The existence of a solution to the above problem can be established via the LeraySchauder fixed point theorem ([5], p.280). To this end, we define an operator $\mathbb{T}: L^{\infty}\left(\Omega_{T}\right) \rightarrow L^{\infty}\left(\Omega_{T}\right)$ as follows: Let $U \in L^{\infty}\left(\Omega_{T}\right)$. We say $w=\mathbb{T}(U)$ if $w$ is the unique solution of the problem

$$
\begin{aligned}
\partial_{t} w-m \operatorname{div}\left(\left(U^{+}+\sigma\right)^{m-1} \nabla w\right) & =-\operatorname{div}\left(\left(U^{+}\right)^{q} \nabla V\right) \text { in } \Omega_{T}, \\
\frac{\partial w}{\partial \mathbf{n}} & =0 \text { on } \Sigma_{T}, \\
\left.w\right|_{t=0} & =u_{0} \text { on } \Omega,
\end{aligned}
$$

where $V$ solves the problem

$$
\begin{aligned}
\partial_{t} V-\Delta V+V & =U \text { in } \Omega_{T}, \\
\frac{\partial V}{\partial \mathbf{n}} & =0 \text { on } \Sigma_{T}, \\
\left.V\right|_{t=0} & =v_{0} \text { on } \Omega .
\end{aligned}
$$

To see that $\mathbb{T}$ is well-defined, we conclude from Proposition 2.3 that $|\nabla V| \in L^{\infty}\left(\Omega_{T}\right)$. Moreover, the two initial boundary value problems in the definition of $\mathbb{T}$ are both linear and uniformly parabolic. We can infer from ([14], Chap. III) that $w$ is Hölder continuous in $\overline{\Omega_{T}}$. It follows that $\mathbb{T}$ is continuous and maps bounded sets into precompact ones. We still need to show that there is a positive number c such that

$$
\|U\|_{\infty, \Omega_{T}} \leq c
$$

for all $U \in L^{\infty}\left(\Omega_{T}\right)$ and $\eta \in(0,1)$ satisfying $U=\eta \mathbb{T}(U)$. This equation is equivalent to the following problem

$$
\begin{aligned}
\partial_{t} U-m \operatorname{div}\left(\left(U^{+}+\sigma\right)^{m-1} \nabla U\right) & =-\eta \operatorname{div}\left(\left(U^{+}\right)^{q} \nabla V\right) \text { in } \Omega_{T}, \\
\partial_{t} V-\Delta V+V & =U \text { in } \Omega_{T}, \\
\frac{\partial U}{\partial \mathbf{n}} & =\frac{\partial V}{\partial \mathbf{n}}=0 \text { on } \Sigma_{T}, \\
\left.(U, V)\right|_{t=0} & =\left(\eta u_{0}, v_{0}\right) \text { on } \Omega .
\end{aligned}
$$


Use $U^{-}$as a test function in (3.6) to get

$$
-\frac{1}{2} \frac{d}{d t} \int_{\Omega}\left(U^{-}\right)^{2} d x-m \int_{\Omega}\left(U^{+}+\sigma\right)^{m-1}\left|\nabla U^{-}\right|^{2} d x=0 .
$$

Integrate to get

$$
U \geq 0 \text { a.e. on } \Omega_{T} \text {. }
$$

This implies that

$$
V \geq 0 \text { a.e. on } \Omega_{T} \text {. }
$$

We introduce the following change of dependent variables

$$
u=U+\sigma, \quad v=V+\sigma .
$$

Then $(u, v)$ satisfies the problem

$$
\begin{aligned}
u_{t}-\Delta u^{m} & \left.=-\eta \operatorname{div}(u-\sigma)^{q} \nabla v\right) \text { in } \Omega_{T}, \\
v_{t}-\Delta v+v & =u \text { in } \Omega_{T}, \\
\frac{\partial u}{\partial \mathbf{n}} & =\frac{\partial v}{\partial \mathbf{n}}=0 \text { on } \Sigma_{T}, \\
\left.(u, v)\right|_{t=0} & =\left(\eta u_{0}(x)+\sigma, v_{0}(x)+\sigma\right) \text { on } \Omega .
\end{aligned}
$$

There is no loss of generality for us to assume that $T \leq 1$. Otherwise, we simply consider $(u(x, T t), v(x, T t))$ on $[0,1]$. From here on we will do that, and also let

$$
\sigma \in(0,1) .
$$

We already have $\eta \in(0,1)$. The generic positive number $c$ will be independent of all three of them.

Lemma 3.1. Let (H3) hold. Then for each s sufficiently large there is a positive number c such that

$$
\sup _{0 \leq t \leq T} \int_{\Omega} u^{s+1} d x+\int_{\Omega_{T}}\left|\nabla u^{\frac{m+s}{2}}\right|^{2} d x d t \leq c\|\nabla v\|_{\infty, \Omega_{T}}^{\frac{m+s}{m-q}}+c .
$$

Proof. First remember that

$$
\sigma \leq u \in L^{\infty}\left(\Omega_{T}\right)
$$

Thus for each $r \in \mathbb{R}$, we have

$$
u^{r} \in L^{2}\left(0, T ; W^{1,2}(\Omega)\right) .
$$

Now pick a number

$$
s>\max \{0, m-2 q\} .
$$

Use $u^{s}$ as a test function in (3.13) to derive

$$
\begin{aligned}
& \frac{1}{s+1} \frac{d}{d t} \int_{\Omega} u^{s+1} d x+m s \int_{\Omega} u^{m+s-2}|\nabla u|^{2} d x \\
& =s \eta \int_{\Omega}(u-\sigma)^{q} u^{s-1} \nabla v \nabla u d x \leq s \int_{\Omega} u^{q+s-1}|\nabla v \nabla u| d x \\
& \leq \frac{1}{2} m s \int_{\Omega} u^{m+s-2}|\nabla u|^{2} d x+\frac{s\|\nabla v\|_{\infty, \Omega_{T}}^{2}}{2 m} \int_{\Omega} u^{2 q-m+s} d x .
\end{aligned}
$$

To estimate the last integral, first notice that

$$
\int_{\Omega} u^{m+s-2}|\nabla u|^{2} d x=\frac{4}{(m+s)^{2}} \int_{\Omega}\left|\nabla u^{\frac{m+s}{2}}\right|^{2} d x .
$$


Recall the Sobolev embedding theorem which states that for each $r \in[1, N)$ there is a positive number $c$ such that

$$
\|w\|_{\frac{N r}{N-r}, \Omega} \leq c\|\nabla w\|_{r, \Omega}+c\|w\|_{1, \Omega} \text { for each } w \in W^{1, r}(\Omega) .
$$

We wish to apply this inequality with $w=u^{\frac{m+s}{2}}$ and $r=2$. For this purpose, we further require

$$
\frac{2 q-m+s}{m+s} \leq \frac{N}{N-2}
$$

or equivalently,

$$
s \geq \frac{(2 q-m)(N-2)-N m}{2} .
$$

We derive from Hölder's inequality and (3.24) that

$$
\begin{aligned}
\int_{\Omega} u^{2 q-m+s} d x & =\int_{\Omega}\left(u^{m+s}\right)^{\frac{2 q-m+s}{m+s}} d x \\
& \leq c\left(\int_{\Omega}\left(u^{m+s}\right)^{\frac{N}{N-2}} d x\right)^{\frac{(2 q-m+s)(N-2)}{(m+s) N}} \\
& \leq c\left(\int_{\Omega}\left|\nabla u^{\frac{m+s}{2}}\right|^{2} d x+\left(\int_{\Omega} u^{\frac{m+s}{2}} d x\right)^{2}\right)^{\frac{2 q-m+s}{m+s}} \\
& \leq c\left(\int_{\Omega}\left|\nabla u^{\frac{m+s}{2}}\right|^{2} d x\right)^{\frac{2 q-m+s}{m+s}}+c\left(\int_{\Omega} u^{\frac{m+s}{2}} d x\right)^{\frac{2(2 q-m+s)}{m+s}} .
\end{aligned}
$$

We integrate (3.13) over $\Omega$ to get

$$
\frac{d}{d t} \int_{\Omega} u d x=0
$$

Subsequently,

$$
\int_{\Omega} u(x, t) d x=\int_{\Omega}\left(\eta u_{0}(x)+\sigma\right) d x \leq c \text { for each } t>0 .
$$

If we further assume that

$$
\frac{m+s}{2}<2 q-m+s,
$$

then we can appeal to the interpolation inequality ([5], p.146), thereby deriving

$$
\|u\|_{\frac{m+s}{2}, \Omega} \leq \varepsilon\|u\|_{2 q-m+s, \Omega}+\frac{1}{\varepsilon^{\mu}}\|u\|_{1, \Omega} \leq \varepsilon\|u\|_{2 q-m+s, \Omega}+\frac{c}{\varepsilon^{\mu}},
$$

where $\varepsilon>0, \mu=\left(1-\frac{2}{m+s}\right) /\left(\frac{2}{m+s}-\frac{1}{2 q-m+s}\right)$. Condition (3.29) is equivalent to

$$
s>3 m-4 q .
$$

Use (3.30) in (3.27) and choose $\varepsilon$ suitably small in the resulting inequality to obtain

$$
\int_{\Omega} u^{2 q-m+s} d x \leq c\left(\int_{\Omega}\left|\nabla u^{\frac{m+s}{2}}\right|^{2} d x\right)^{\frac{2 q-m+s}{m+s}}+c .
$$


Plug this into (3.22) to get

$$
\begin{aligned}
& \frac{1}{s+1} \frac{d}{d t} \int_{\Omega} u^{s+1} d x+\frac{2 m s}{(m+s)^{2}} \int_{\Omega}\left|\nabla u^{\frac{m+s}{2}}\right|^{2} d x \\
& \leq c\left(\int_{\Omega}\left|\nabla u^{\frac{m+s}{2}}\right|^{2} d x\right)^{\frac{2 q-m+s}{m+s}}\|\nabla v\|_{\infty, \Omega_{T}}^{2}+c\|\nabla v\|_{\infty, \Omega_{T}}^{2} \\
& \leq \varepsilon \int_{\Omega}\left|\nabla u^{\frac{m+s}{2}}\right|^{2} d x+c(\varepsilon)\|\nabla v\|_{\infty, \Omega_{T}}^{\frac{m+s}{m-q}}+c\|\nabla v\|_{\infty, \Omega_{T}}^{2} .
\end{aligned}
$$

The last step is due to the assumption $m>q$ and Young's inequality ([5], p. 145). Once again, by taking $\varepsilon$ suitably small, we arrive at

$$
\sup _{0 \leq t \leq T} \int_{\Omega} u^{s+1} d x+\int_{\Omega_{T}}\left|\nabla u^{\frac{m+s}{2}}\right|^{2} d x d t \leq c\|\nabla v\|_{\infty, \Omega_{T}}^{\frac{m+s}{m-q}} d t+c .
$$

Here we have used the fact $\frac{m+s}{m-q}>2$ due to (3.21). That is to say, the lemma is valid for any $s$ that satisfies (3.21), (3.26), and (3.31). This completes the proof.

Lemma 3.2. Let (H3) hold and $s$ be given as in Lemma 3.1. Then there is a positive number $c$ such that

$$
\|u\|_{\infty, \Omega_{T}} \leq c\|\nabla v\|_{\infty, \Omega_{T}}^{\gamma}+c
$$

where

$$
\gamma=\frac{\left[(s+1)(N+2)+N(m-1)^{+}\right](m+s)+(s+1) N(m-q)(N+2)}{(s+1)(m-q)[(N+2)(s+1)+2 N(m-q)]} .
$$

Proof. Let

$$
K \geq 2\left(\left\|u_{0}\right\|_{\infty, \Omega}+1\right)
$$

be selected as below. Define

$$
K_{n}=K-\frac{K}{2^{n+1}}, n=0,1 \cdots
$$

Obviously,

$$
\frac{K}{2} \leq K_{n} \leq K
$$

Set

$$
\begin{aligned}
S_{n}(t) & =\left\{x \in \Omega: u(x, t) \geq K_{n}\right\}, \\
A_{n} & =\cup_{0 \leq t \leq T} S_{n}(t)=\left\{(x, t) \in \Omega_{T}: u(x, t) \geq K_{n}\right\} .
\end{aligned}
$$

Subsequently,

$$
\int_{0}^{T}\left|S_{n+1}(t)\right| d t=\left|A_{n+1}\right|
$$

To simplify our presentation, we also introduce two parameters

$$
\begin{aligned}
m_{s} & =(s+1) \frac{2}{N}+m+s, \\
q_{s} & =m_{s}-(2 q-m+s),
\end{aligned}
$$


where $s$ is given as in Lemma 3.1, i.e., $s$ is sufficiently large. Then use $\left(u^{s}-K_{n+1}^{s}\right)^{+}$as a test function in (3.13) to derive

$$
\begin{aligned}
& \frac{d}{d t} \int_{\Omega} \int_{0}^{u}\left(\tau^{s}-K_{n+1}^{s}\right)^{+} d \tau d x+m s \int_{S_{n+1}(t)} u^{m+s-2}|\nabla u|^{2} d x \\
& \quad=s \eta \int_{S_{n+1}(t)}(u-\sigma)^{q} u^{s-1} \nabla v \nabla u d x \leq s \int_{S_{n+1}(t)} u^{q+s-1}|\nabla v||\nabla u| d x .
\end{aligned}
$$

After a suitable application of Cauchy's inequality ([14], p. 58), we integrate to obtain

$$
\begin{aligned}
& \sup _{0 \leq t \leq T} \int_{\Omega} \int_{0}^{u}\left(\tau^{s}-K_{n+1}^{s}\right)^{+} d \tau d x+\int_{A_{n+1}}\left|\nabla u^{\frac{m+s}{2}}\right|^{2} d x d t \\
& \quad \leq c \int_{A_{n+1}} u^{2 q-m+s}|\nabla v|^{2} d x d t \leq c\|\nabla v\|_{\infty, \Omega_{T}}^{2} \int_{A_{n+1}} u^{2 q-m+s} d x d t
\end{aligned}
$$

Since $s>1$, we have

$$
\begin{aligned}
\int_{K_{n+1}}^{u}\left(\tau^{s}-K_{n+1}^{s}\right)^{+} d \tau \chi_{A_{K_{n+1}}} & \geq \int_{K_{n+1}}^{u}\left[\left(\tau-K_{n+1}\right)^{+}\right]^{s} d \tau \\
& =\frac{1}{s+1}\left[\left(u-K_{n+1}\right)^{+}\right]^{s+1}
\end{aligned}
$$

Recall that $m_{s}=(s+1) \frac{2}{N}+m+s$. We estimate, with the aid of Hölder's inequality and (3.24), that

$$
\begin{aligned}
y_{n+1} \equiv & \int_{0}^{T} \int_{\Omega}\left[\left(u-K_{n+1}\right)^{+}\right]^{m_{s}} d x d t \\
\leq & \int_{0}^{T}\left(\int_{\Omega}\left[\left(u-K_{n+1}\right)^{+}\right]^{s+1} d x\right)^{\frac{2}{N}}\left(\int_{\Omega}\left[\left(u-K_{n+1}\right)^{+}\right]^{\frac{(m+s) N}{N-2}}\right)^{\frac{N-2}{N}} d t \\
\leq & c\left(\sup _{0 \leq t \leq T} \int_{\Omega}\left[\left(u-K_{n+1}\right)^{+}\right]^{s+1} d x\right)^{\frac{2}{N}} \\
& \cdot \int_{0}^{T}\left(\int_{\Omega}\left|\nabla\left[\left(u-K_{n+1}\right)^{+}\right]^{\frac{m+s}{2}}\right|^{2} d x+\left(\int_{\Omega}\left[\left(u-K_{n+1}\right)^{+}\right]^{\frac{m+s}{2}} d x\right)^{2}\right) d t
\end{aligned}
$$

We can easily verify that

$$
\begin{aligned}
\left|\nabla\left[\left(u-K_{n+1}\right)^{+}\right]^{\frac{m+s}{2}}\right| & =\frac{m+s}{2}\left[\left(u-K_{n+1}\right)^{+}\right]^{\frac{m+s}{2}-1}|\nabla u| \\
& \leq \frac{m+s}{2} u^{\frac{m+s}{2}-1}|\nabla u| \chi_{S_{n+1}(t)}=\left|\nabla u^{\frac{m+s}{2}}\right| \chi_{S_{n+1}(t)}, \\
\int_{\Omega}\left[\left(u-K_{n+1}\right)^{+}\right]^{\frac{m+s}{2}} d x & \leq\left(\int_{\Omega}\left[\left(u-K_{n+1}\right)^{+}\right]^{m_{s}} d x\right)^{\frac{m+s}{2 m_{s}}}\left|S_{n+1}(t)\right|^{1-\frac{m+s}{2 m_{s}}} .
\end{aligned}
$$


The latter yields

$$
\begin{aligned}
& \int_{0}^{T}\left(\int_{\Omega}\left[\left(u-K_{n+1}\right)^{+}\right]^{\frac{m+s}{2}} d x\right)^{2} d t \\
& \leq \int_{0}^{T}\left(\int_{\Omega}\left[\left(u-K_{n+1}\right)^{+}\right]^{m_{s}} d x\right)^{\frac{m+s}{m_{s}}}\left|S_{n+1}(t)\right|^{2-\frac{m+s}{m_{s}}} d t \\
& \leq\left(\int_{\Omega_{T}}\left[\left(u-K_{n+1}\right)^{+}\right]^{m_{s}} d x d t\right)^{\frac{m+s}{m_{s}}}\left(\int_{0}^{T}\left|S_{n+1}(t)\right|^{1+\frac{N m_{s}}{2(s+1)}} d t\right)^{\frac{2(s+1)}{N m_{s}}} \\
& \leq c\left|A_{n+1}\right|^{\frac{2(s+1)}{N m_{s}}} y_{n}^{\frac{m+s}{m_{s}}} .
\end{aligned}
$$

Here we have used the fact that $\left\{y_{n}\right\}$ is a decreasing sequence. Use (3.49) and (3.51) in (3.48) and take (3.46) into account to derive

$$
\begin{aligned}
y_{n+1} \leq & c\|\nabla v\|_{\infty, \Omega_{T}}^{\frac{2(N+2)}{N}}\left(\int_{A_{n+1}} u^{2 q-m+s} d x d t\right)^{\frac{N+2}{N}} \\
& +c\|\nabla v\|_{\infty, \Omega_{T}}^{\frac{4}{N}}\left(\int_{A_{n+1}} u^{2 q-m+s} d x d t\right)^{\frac{2}{N}}\left|A_{n+1}\right|^{\frac{2(s+1)}{N m_{s}}} y_{n}^{\frac{m+s}{m_{s}}} .
\end{aligned}
$$

The first integral on the right-hand side of (3.52) can be estimated as follows:

$$
\begin{aligned}
\left(\int_{A_{n+1}} u^{2 q-m+s} d x d t\right)^{\frac{N+2}{N}} & =K_{n+1}^{\frac{(N+2)(2 q-m+s)}{N}}\left(\int_{A_{n+1}}\left(\frac{u}{K_{n+1}}\right)^{2 q-m+s} d x d t\right)^{\frac{N+2}{N}} \\
& \leq \frac{1}{K_{n+1}^{\frac{(N+2) m_{s}}{N}-\frac{(N+2)(2 q-m+s)}{N}}}\left(\int_{A_{n+1}} u^{m_{s}} d x d t\right)^{\frac{N+2}{N}} \\
& =\frac{1}{K_{n+1}^{\frac{(N+2) q_{s}}{N}}}\left(\int_{A_{n+1}} u^{m_{s}} d x d t\right)^{1+\frac{2}{N}} .
\end{aligned}
$$

Similarly,

$$
\left(\int_{A_{n+1}} u^{2 q-m+s} d x d t\right)^{\frac{2}{N}} \leq \frac{1}{K_{n+1}^{\frac{2 q_{s}}{N}}}\left(\int_{A_{n+1}} u^{m_{s}} d x d t\right)^{\frac{2}{N}} .
$$

Recall that

$$
K_{n+1}-K_{n}=\frac{K}{2^{n+2}}, \quad \frac{K_{n+1}-K_{n}}{K_{n+1}}=\frac{1}{2^{n+2}-1}>\frac{1}{2^{n+2}} .
$$

With the aid of the preceding two results, we obtain

$$
\begin{aligned}
y_{n} & \geq \int_{A_{n+1}}\left[\left(K_{n+1}-K_{n}\right)^{+}\right]^{m_{s}} d x d t=\frac{K^{m_{s}}}{2^{(n+2) m_{s}}}\left|A_{n+1}\right| \\
y_{n} & \geq \int_{A_{n+1}} u^{m_{s}}\left[\left(1-\frac{K_{n}}{u}\right)^{+}\right]^{m_{s}} d x d t \\
& \geq \int_{A_{n+1}} u^{m_{s}}\left(1-\frac{K_{n}}{K_{n+1}}\right)^{m_{s}} d x d t \geq \frac{1}{2^{(n+2) m_{s}}} \int_{A_{n+1}} u^{m_{s}} d x d t .
\end{aligned}
$$


By (3.55),

$$
\left|A_{n+1}\right|^{\frac{2(s+1)}{N m_{s}}} \leq \frac{2^{\frac{2(n+2)(s+1)}{N}}}{K^{\frac{2(s+1)}{N}}} y_{n}^{\frac{2(s+1)}{N m_{s}}} .
$$

Keeping this, (3.54), (3.39), and (3.56) in mind, we derive from (3.52) that

$$
\begin{aligned}
y_{n+1} \leq & \frac{c\|\nabla v\|_{\infty, \Omega_{T}}^{\frac{2(N+2)}{N}} 2^{\frac{(n+2) m_{s}(N+2)}{N}}}{K_{n+1}^{\frac{(N+2) q_{s}}{N}}} y_{n}^{1+\frac{2}{N}} \\
& +\frac{c 2^{\frac{\left[2(s+1)+2 m_{s}\right](n+2)}{N}}\|\nabla v\|_{\infty, \Omega_{T}}^{\frac{4}{N}} y_{n}^{1+\frac{2}{N}}}{K^{\frac{2(s+1)}{N}+\frac{2 q_{s}}{N}}} \\
\leq & c b^{n}\left(\frac{\|\nabla v\|_{\infty, \Omega_{T}}^{\frac{2(N+2)}{N}}}{K^{\frac{(N+2) q_{s}}{N}}}+\frac{\|\nabla v\|_{\infty, \Omega_{T}}^{\frac{4}{N}}}{K^{\frac{2(s+1)+2 q_{s}}{N}}}\right) y_{n}^{1+\frac{2}{N}}
\end{aligned}
$$

where

$$
b=\max \left\{2^{\frac{m_{s}(N+2)}{N}}, 2^{\frac{2(s+1)+2 m_{s}}{N}}\right\} .
$$

We can easily check from (3.43) and (3.44) that

$$
(N+2) q_{s} \geq 2(s+1)+2 q_{s} \text { if and only if } m \geq q .
$$

Recall that $K_{n} \geq 1$. Thus if (H3) holds, we can deduce from (3.57) that

$$
y_{n+1} \leq \frac{c b^{n}\left(\|\nabla v\|_{\infty, \Omega_{T}}^{\frac{2(N+2)}{N}}+\|\nabla v\|_{\infty, \Omega_{T}}^{\frac{4}{N}}\right)}{K^{\frac{2(s+1)+2 q_{s}}{N}}} y_{n}^{1+\frac{2}{N}} .
$$

According to Proposition 2.1, if we choose $K$ so large that

$$
\begin{aligned}
y_{0} & =\int_{\Omega_{T}}\left[\left(u-\frac{K}{2}\right)^{+}\right]^{m_{s}} d x d t \leq \int_{\Omega_{T}} u^{m_{s}} d x d t \\
& \leq \frac{c K^{s+1+q_{s}}}{\|\nabla v\|_{\infty, \Omega_{T}}^{N+2}+\|\nabla v\|_{\infty, \Omega_{T}}^{2}},
\end{aligned}
$$

then

$$
\sup _{\Omega_{T}} u \leq K
$$

In view of (3.37), it is enough for us to take

$$
\begin{aligned}
K= & c\left(\int_{\Omega_{T}} u^{m_{s}} d x d t\right)^{\frac{1}{s+1+q_{s}}}\left(\|\nabla v\|_{\infty, \Omega_{T}}^{N+2}+\|\nabla v\|_{\infty, \Omega_{T}}^{2}\right)^{\frac{1}{s+1+q_{s}}} \\
& +2\left\|u_{0}\right\|_{\infty, \Omega}+2 .
\end{aligned}
$$


In light of (3.48), (3.51), and (3.18), we have

$$
\begin{aligned}
\int_{\Omega_{T}} u^{m_{s}} d x d t \leq & \left(\sup _{0 \leq t \leq T} \int_{\Omega} u^{s+1} d x\right)^{\frac{2}{N}} \int_{\Omega_{T}}\left|\nabla u^{\frac{m+s}{2}}\right|^{2} d x d t \\
& +c\left(\sup _{0 \leq t \leq T} \int_{\Omega} u^{s+1} d x\right)^{\frac{2}{N}}\left(\int_{\Omega_{T}} u^{m_{s}} d x d t\right)^{\frac{m+s}{m_{s}}} \\
\leq & c\|\nabla v\|_{\infty, \Omega_{T}}^{\frac{(N+2)(m+s)}{N(m-q)}}+c+\varepsilon \int_{\Omega_{T}} u^{m_{s}} d x d t+c(\varepsilon)\left(\sup _{0 \leq t \leq T} \int_{\Omega} u^{s+1} d x\right)^{\frac{m_{s}}{s+1}} .
\end{aligned}
$$

Choosing $\varepsilon$ suitably small, we arrive at

$$
\int_{\Omega_{T}} u^{m_{s}} d x d t \leq c\|\nabla v\|_{\infty, \Omega_{T}}^{\frac{(N+2)(m+s)}{N(m-q)}}+c\|\nabla v\|_{\infty, \Omega_{T}}^{\frac{m_{s}(m+s)}{(s+1)(m-q)}}+c .
$$

Substituting this into (3.58) yields

$$
\begin{aligned}
\|u\|_{\infty, \Omega_{T}} & \leq c\left[\left(\|\nabla v\|_{\infty, \Omega_{T}}^{\frac{(N+2)(m+s)}{N(m-q)}}+\|\nabla v\|_{\infty, \Omega_{T}}^{\frac{m_{s}(m+s)}{(s+1)(m-q)}}+1\right)\left(\|\nabla v\|_{\infty, \Omega_{T}}^{N+2}+\|\nabla v\|_{\infty, \Omega_{T}}^{2}\right)\right]^{\frac{1}{s+1+q_{s}}}+c \\
& \leq c\left[\left(\|\nabla v\|_{\infty, \Omega_{T}}^{\left(\frac{N+2}{N}+\frac{(m-1)+}{s+1}\right) \frac{m+s}{m-q}}+1\right)\left(\|\nabla v\|_{\infty, \Omega_{T}}^{N+2}+1\right)\right]^{\frac{1}{s+1+q_{s}}}+c \\
& \leq c\left(\|\nabla v\|_{\infty, \Omega_{T}}^{\frac{\left[(s+1)(N+2)+N(m-1)^{+}\right](m+s)}{(s+1) N(m-q)}+N+2}+1\right)^{\frac{1}{s+1+q_{s}}}+c \\
& \leq c\|\nabla v\|_{\infty, \Omega_{T}}^{\frac{\left[(s+1)(N+2)+N(m-1)^{+}\right](m+s)+(s+1) N(m-q)(N+2)}{(s+1) N(m-q)\left(s+1+q_{s}\right)}}+c .
\end{aligned}
$$

This together with (3.44) implies the lemma.

Proof of Theorem 1.2 under (Н3). We wish to show

$$
\|v\|_{L^{\infty}\left(0, T ; W^{1, \infty}(\Omega)\right)}+\|u\|_{\infty, \Omega \times[0, T]} \leq c .
$$

Let $\gamma$ be given as in Lemma 3.2. Note that

$$
\lim _{s \rightarrow \infty} \gamma=\lim _{s \rightarrow \infty} \frac{\left[(s+1)(N+2)+N(m-1)^{+}\right](m+s)+(s+1) N(m-q)(N+2)}{(s+1)(m-q)[(N+2)(s+1)+2 N(m-q)]}=\frac{1}{m-q} .
$$

If $\frac{1}{m-q}>1$, then there is a $\beta>0$ such that

$$
\gamma=1+\beta \text { for some suitably large } s .
$$

Fix this $s$ and let $p$ be given as in Proposition 2.3. We can derive from (2.9) and Lemma 3.2

$$
\begin{aligned}
\|v\|_{L^{\infty}\left(0, T ; W^{1, \infty}(\Omega)\right)}+\|u\|_{\infty, \Omega_{T}} & \leq c\|u\|_{2 p, \Omega_{T}}+c\|\nabla v\|_{\infty, \Omega_{T}}^{1+\beta}+c \\
& \leq c T^{\frac{1}{2 p}}\|u\|_{\infty, \Omega_{T}}+c\|u\|_{2 p, \Omega_{T}}^{1+\beta}+c \\
& \leq c T^{\frac{1}{2 p}}\|\nabla v\|_{\infty, \Omega_{T}}^{1+\beta}+c T^{\frac{1+\beta}{2 p}}\|u\|_{\infty, \Omega_{T}}^{1+\beta}+c \\
& \leq c T^{\frac{1}{2 p}}\left(\|v\|_{L^{\infty}\left(0, T ; W^{1, \infty}(\Omega)\right)}+\|u\|_{\infty, \Omega_{T}}\right)^{1+\beta}+c .
\end{aligned}
$$

Here we have used the fact that $T \leq 1$. Set

$$
h(\tau)=\|v\|_{L^{\infty}\left(0, \tau ; W^{1, \infty}(\Omega)\right)}+\|u\|_{\infty, \Omega \times[0, \tau]} .
$$


Let $T_{0} \in(0, T]$ be selected below. It follows from (3.61) that

$$
h(\tau) \leq c T_{0}^{\frac{1}{2 p}} h^{1+\beta}(\tau)+c \text { for each } \tau \in\left[0, T_{0}\right] .
$$

It is not difficult for us to see from the proof of Proposition 2.3 that $\nabla v$ is actually Hölder continuous on $\overline{\Omega_{T}}$, so is $u$ for each fixed $\sigma>0$. Thus $h(\tau)$ is a continuous function of $\tau$. In view of Proposition 2.2 , if we choose $T_{0}$ so that

$$
c T_{0}^{\frac{1}{2 p}} \leq \frac{\beta^{\beta}}{(c+\beta)^{\beta}(1+\beta)^{1+\beta}} \text { and }\left\|\nabla\left(v_{0}+1\right)\right\|_{W^{1, \infty}(\Omega)}+\left\|u_{0}+1\right\|_{\infty, \Omega} \leq \frac{1}{\left[c T_{0}^{\frac{1}{2 p}}(1+\beta)\right]^{\frac{1}{\beta}}}
$$

then

$$
\|\nabla v\|_{\infty, \Omega \times\left[0, T_{0}\right]} \leq \frac{1}{\left[c T_{0}^{\frac{1}{2 p}}(1+\beta)\right]^{\frac{1}{\beta}}} .
$$

By setting $T=0$ in $(3.61)$, we see that $\left\|\nabla\left(v_{0}+1\right)\right\|_{W^{1, \infty}(\Omega)}+\left\|u_{0}+1\right\|_{\infty, \Omega} \leq c$. If we take

$$
c T_{0}^{\frac{1}{2 p}}=\frac{\beta^{\beta}}{(c+\beta)^{\beta}(1+\beta)^{1+\beta}},
$$

then the second inequality in (3.64) is automatically satisfied. Upon doing so, we arrive at

$$
\|v\|_{L^{\infty}\left(0, T_{0} ; W^{1, \infty}(\Omega)\right)}+\|u\|_{\infty, \Omega \times\left[0, T_{0}\right]} \leq \frac{(c+\beta)(1+\beta)}{\beta} .
$$

Set $k=\left\lfloor\frac{T}{T_{0}}\right\rfloor$, the integer part of the number $\frac{T}{T_{0}}$. If $k \geq 1$, we consider

$$
u_{T_{0}}(x, t)=u\left(t+T_{0}, x\right), \quad v_{T_{0}}(x, t)=v\left(t+T_{0}, x\right) \text { on }\left[0, T_{0}\right] .
$$

Obviously, $\left(u_{T_{0}}, v_{T_{0}}\right)$ satisfies the same conditions as $(u, v)$ does on $\Omega \times\left(0, T_{0}\right)$. Thus we can repeat the previous arguments to yield (3.67) for $\left(u_{T_{0}}, v_{T_{0}}\right)$. After a finite number of steps, we obtain (3.59). Of course, in the last step, we will have to use $\min \left\{T_{0}, T-k T_{0}\right\}$ instead of $T_{0}$.

If $\frac{1}{m-q}<1$, an application of Young's inequality is enough to reach (3.59).

If $\frac{1}{m-q}=1$, this can also be handled easily. We verify that $\frac{d \gamma}{d s}$ changes signs at most three times. Thus either $\gamma$ decreases toward 1 as $s \rightarrow \infty$, which can be treated like the first case, or $\gamma$ increases toward 1 as $s \rightarrow \infty$, which is essentially the second case.

Clearly, (3.5) is a consequence of (3.59). Thus we can conclude from the Leray-Schauder fixed point theorem that (3.1)-(3.4) has a solution. Denote the solution by $\left(U_{\sigma}, V_{\sigma}\right)$. In view of (3.10), we can rewrite (3.1)-(3.4) as

$$
\begin{aligned}
\partial_{t} U_{\sigma}-m \operatorname{div}\left(\left(U_{\sigma}+\sigma\right)^{m-1} \nabla U_{\sigma}\right) & =-\operatorname{div}\left(U_{\sigma}^{q} \nabla V_{\sigma}\right) \text { in } \Omega_{T}, \\
\partial_{t} V_{\sigma}-\Delta V_{\sigma}+V_{\sigma} & =U_{\sigma} \text { in } \Omega_{T}, \\
\frac{\partial U_{\sigma}}{\partial \mathbf{n}} & =\frac{\partial V_{\sigma}}{\partial \mathbf{n}}=0 \text { on } \Sigma_{T}, \\
\left.\left(U_{\sigma}, V_{\sigma}\right)\right|_{t=0} & =\left(u_{0}, v_{0}\right) \text { on } \Omega .
\end{aligned}
$$

Furthermore,

$$
U_{\sigma} \geq 0, \quad V_{\sigma} \geq 0, \quad \text { and }\left\|V_{\sigma}\right\|_{L^{\infty}\left(0, T ; W^{1, \infty}(\Omega)\right)}+\left\|U_{\sigma}\right\|_{\infty, \Omega_{T}} \leq c .
$$

We wish to show that we can take $\sigma \rightarrow 0$ in (3.69)-(3.72). For this purpose, we use $\left(U_{\sigma}+\sigma\right)^{m}$ as a test function in (3.69) to derive

$$
\frac{1}{m+1} \sup _{0 \leq t \leq T} \int_{\Omega}\left(U_{\sigma}+\sigma\right)^{m+1} d x+\int_{\Omega_{T}}\left|\nabla\left(U_{\sigma}+\sigma\right)^{m}\right|^{2} d x d t \leq c .
$$


We compute

$$
\begin{aligned}
\partial_{t}\left(U_{\sigma}+\sigma\right)^{m+1}= & (m+1)\left(U_{\sigma}+\sigma\right)^{m} \partial_{t} U_{\sigma} \\
= & (m+1) \operatorname{div}\left(\left(U_{\sigma}+\sigma\right)^{m} \nabla\left(U_{\sigma}+\sigma\right)^{m}\right)-(m+1)\left|\nabla\left(U_{\sigma}+\sigma\right)^{m}\right|^{2} \\
& -(m+1) \operatorname{div}\left(\left(U_{\sigma}+\sigma\right)^{m} U_{\sigma}^{q} \nabla V_{\sigma}\right)+(m+1) U_{\sigma}^{q} \nabla V_{\sigma} \cdot \nabla\left(U_{\sigma}+\sigma\right)^{m}, \\
\nabla\left(U_{\sigma}+\sigma\right)^{m+1}= & \frac{m+1}{m}\left(U_{\sigma}+\sigma\right) \nabla\left(U_{\sigma}+\sigma\right)^{m} .
\end{aligned}
$$

Thus the sequence $\left\{\partial_{t}\left(U_{\sigma}+\sigma\right)^{m+1}\right\}$ is bounded in $L^{2}\left(0, T ;\left(W^{1,2}(\Omega)\right)^{*}\right)+L^{1}\left(\Omega_{T}\right) \equiv\left\{\psi_{1}+\psi_{2}: \psi_{1} \in\right.$ $\left.L^{2}\left(0, T ;\left(W^{1,2}(\Omega)\right)^{*}\right), \psi_{2} \in L^{1}\left(\Omega_{T}\right)\right\}$ and the sequence $\left\{\left(U_{\sigma}+\sigma\right)^{m+1}\right\}$ is bounded in $L^{2}\left(0, T ; W^{1,2}(\Omega)\right)$. This puts us in a position to apply the Lions-Aubin lemma [18]. Upon doing so, we obtain the precompactness of $\left\{\left(U_{\sigma}+\sigma\right)^{m+1}\right\}$ in $L^{2}\left(\Omega_{T}\right)$. We can extract a subsequence of $\left\{U_{\sigma}+\sigma\right\}$, still denoted by $\left\{U_{\sigma}+\sigma\right\}$, such that $U_{\sigma}+\sigma$ converges a.e. on $\Omega_{T}$. This is enough to justify passing to the limit in (3.69)-(3.72). The proof is complete.

We would like to remark that as $m \rightarrow q^{+}$the upper bound in (3.67) deteriorates. This foretells the possibility that solutions blow up if $m=q$.

Proof of Theorem 1.2 under (H4). We will show that an estimate like (3.35) remains true even without the benefit of Lemma 3.1. Let $s$ be given as before, i.e., $s$ is large enough. With the aid of (H4), we can derive from (3.57) that

$$
y_{n+1} \leq \frac{c b^{n}\left(\|\nabla v\|_{\infty, \Omega_{T}}^{\frac{2(N+2)}{N}}+\|\nabla v\|_{\infty, \Omega_{T}}^{\frac{4}{N}}\right)}{K^{\frac{(N+2) q_{s}}{N}}} y_{n}^{1+\frac{2}{N}} .
$$

In light of Proposition 2.1, if $K$ is so chosen that

$$
y_{0} \leq \frac{c K^{\frac{(N+2) q_{s}}{2}}}{\|\nabla v\|_{\infty, \Omega_{T}}^{N+2}+\|\nabla v\|_{\infty, \Omega_{T}}^{2}}
$$

then

$$
\sup _{\Omega_{T}} u \leq K
$$

In view of $(3.37)$, it is enough for us to take

$$
\begin{aligned}
K= & c\left(\int_{\Omega_{T}} u^{m_{s}} d x d t\right)^{\frac{2}{(N+2) q_{s}}}\left(\|\nabla v\|_{\infty, \Omega_{T}}^{N+2}+\|\nabla v\|_{\infty, \Omega_{T}}^{2}\right)^{\frac{2}{(N+2) q_{s}}} \\
& +2\left\|u_{0}\right\|_{\infty, \Omega}+2
\end{aligned}
$$

If

$$
\frac{2 m_{s}}{(N+2) q_{s}}<1
$$

or equivalently,

then Young's inequality asserts

$$
q<1 \text { and } m>q+\frac{q-1}{N+1}
$$

$$
\begin{aligned}
K \leq & \varepsilon\|u\|_{m_{s}, \Omega_{T}}+c(\varepsilon)\left(\|\nabla v\|_{\infty, \Omega_{T}}^{N+2}+\|\nabla v\|_{\infty, \Omega_{T}}^{2}\right)^{\frac{2}{(N+2) q_{s}-2 m_{s}}} \\
& +2\left\|u_{0}\right\|_{\infty, \Omega}+2
\end{aligned}
$$

Use this in (3.74) to derive

$$
\|u\|_{\infty, \Omega_{T}} \leq c\|\nabla v\|_{\infty, \Omega_{T}}^{\frac{N+2}{(N+1) m-(N+2) q+1}}+c\left\|u_{0}\right\|_{\infty, \Omega}+c .
$$




$$
\frac{2 m_{s}}{(N+2) q_{s}}=1
$$

we can appeal to the interpolation inequality ([5], p. 146) to obtain

$$
\|u\|_{m_{s}, \Omega_{T}} \leq \varepsilon\|u\|_{\infty, \Omega_{T}}+\frac{1}{\varepsilon^{m_{s}-1}}\|u\|_{1, \Omega_{T}} \leq \varepsilon\|u\|_{\infty, \Omega_{T}}+\frac{c}{\varepsilon^{m_{s}-1}} .
$$

With this in mind, we derive from (3.75) that

$$
\begin{aligned}
K \leq & c\left(\varepsilon\|u\|_{\infty, \Omega_{T}}+\frac{c}{\varepsilon^{m_{s}-1}}\right)\left(\|\nabla v\|_{\infty, \Omega_{T}}^{N+2}+\|\nabla v\|_{\infty, \Omega_{T}}^{2}\right)^{\frac{2}{(N+2) q_{s}}} \\
& +2\left\|u_{0}\right\|_{\infty, \Omega}+2 \\
= & \alpha\|u\|_{\infty, \Omega_{T}}+\frac{c}{\alpha^{m_{s}-1}}\left(\|\nabla v\|_{\infty, \Omega_{T}}^{N+2}+\|\nabla v\|_{\infty, \Omega_{T}}^{2}\right)^{\frac{2 m_{s}}{(N+2) q_{s}}} \\
& +2\left\|u_{0}\right\|_{\infty, \Omega}+2 .
\end{aligned}
$$

Plug this into (3.74) and choose $\alpha$ suitably small in the resulting inequality to derive

$$
\|u\|_{\infty, \Omega_{T}} \leq c\|\nabla v\|_{\infty, \Omega_{T}}^{N+2}+c\left\|u_{0}\right\|_{\infty, \Omega}+c .
$$

The rest of the proof is similar to that under (H3). That is, (3.59) can be inferred from either (3.77) or (3.80).

\section{REFERENCES}

[1] M. Burger, M. Di Francesco, and Y. Dolak-Struss, The Keller-Segel model for chemotaxis with prevention of overcrowding: linear vs. nonlinear diffusion, SIAM J. Math. Anal., 38(2006),1288-1315.

[2] V. Calvez and J. A. Carrillo, Volume effects in the Keller-Segel model: energy estimates preventing blow-up, J. Math. Pures Appl., 86(2006), 155-175.

[3] F. Chiarenza, M. Frasca, and P. Longo, $W^{2, p}$-solvability of the Dirichlet problem for nondivergence elliptic equations with VMO coefficients, Trans. Amer. Math. Soc., 336(1993), 841-853.

[4] E. DiBenedetto, Degenerate Parabolic Equations, Springer-Verlag, New York, 1993.

[5] D. Gilbarg and N. S. Trudinger, Elliptic Partial Differential Equations of Second Order, Springer-Verlag, Berlin, 1983.

[6] T. Hillen and K. J. Painter, A user's guide to PDE models for chemotaxis, J. Math. Biol., 58(2009), $183-217$.

[7] S. Hittmeir and A. Jüngel, Cross diffusion preventing blow-up in the two-dimensional Keller-Segel model, SIAM J. Math. Anal., 43(2011), 997-1022.

[8] D. Horstmann, From 1970 until present: The Keller-Segel model in chemotaxis and its consequences, Part I, Jahresbericht der DMV, 105(2003), no. 3, 103-165.

[9] D. Horstmann , From 1970 until present: The Keller-Segel model in chemotaxis and its con- sequences, Part II, Jahresbericht der DMV, 106(2004), no. 2, 51-69.

[10] S. Ishida and T. Yokota, Global existence of weak solutions to quasilinear degenerate Keller-Segel systems of parabolic-parabolic type, J. Differential Equations, 252(2012), 1421-1440.

[11] S. Ishida and T. Yokota, Global existence of weak solutions to quasilinear degenerate Keller-Segel systems of parabolic-parabolic type with small data, J. Differential Equations, 252(2012), 2469-2491.

[12] E.F. Keller and L.A. Segel, Initiation of slime mold aggregation viewed as an instability, J. Theor. Biol., 26 (1970), $399-415$.

[13] S. Kim and Ki-Ahm Lee, Hölder regularity and uniqueness theorem on weak solutions to the degenerate Keller-Segel system, Nonlinear Anal., 138(2016), 229-252.

[14] Q.A. Ladyzenskaja, V.A. Solonnikov, and N.N. Ural'ceva, Linear and Quasi-linear Equations of Parabolic Type, Tran. Math. Monographs, Vol. 23, AMS, Providence, RI, 1968.

[15] C. Miao, Weak solution of class of nonlinear heat equation systems and application to the Navier-Stokes system, J. Differential Equations, 61(1986), 141-151.

[16] C. S. Patlak, Random walk with persistence and external bias, Bull. Math: Biophys. , 15 (1953), 311 - 338.

[17] A.N. Sandjo, S. Moutari, and Y. Gningue, Solutions of fourth-order parabolic equation modeling thin film growth, J. Differential Equations, 259(2015), 7260-7283.

[18] J. Simon, Compact sets in the space $L^{p}(0, T ; B)$, Ann. Mat. Pura Appl., 146(1987), 65-96. 
[19] A. Stevens, The derivation of chemotaxis equations as limit dynamics of moderately interacting stochastic manyparticle systems, SIAM J. Appl. Math., 61(2000), 183-212.

[20] Y. Sugiyama and H. Kunii, Global existence and decay properties for a degenerate Keller-Segel model with a power factor in drift term, J. Differential Equations, 227(2006), 333-364.

[21] X. Xu, Global existence of strong solutions to a groundwater flow problem, arXiv:1912.03793 [math.AP], 2019. Z. Angew. Math. Phys., 71(2020), Art\# 127.

Email address: xxu@math.msstate.edu 